\title{
Development of GNSS Receiver for Mobile CORS with RTK Correction Services Using Cloud Server
}

\author{
Parluhutan Manurung ${ }^{1}$, Hari Pramujo ${ }^{2}$, Joshua BP Manurung ${ }^{2}$ \\ ${ }^{1}$ Badan Informasi Geospasial (BIG) \\ ${ }^{2}$ PT Luwes Inovasi Mandiri
}

\begin{abstract}
Requirements for precise Real Time Kinematic (RTK) positioning have been significantly growing in Indonesia since early 2010, resulting from the national programs on village mapping, land cadastre certification, infrastructure developments, and one map policy. The activities require a subdecimetre level of positioning accuracy to produce detail maps to the scale of 1:500. RTK positioning with CORS should be the most reliable technique to meet the accuracy level. However, the existing distribution of Indonesia CORS Network, consisting of 150 active stations, is still far from adequate since the interstation distances are of around $500 \mathrm{~km}$ while an ideal range should be less than $50 \mathrm{~km}$. This paper related to an initiative for the development of a GNSS receiver that can be transportable and easily set up in the field as a base station to provide RTK CORS correction services with local contents. The initiative supports national efforts on the densification of Indonesia CORS Network to better distribution. The development was started using the Tersus OEM GNSS board series BX305, including the integration of the board to a mini PC. Further developments have been made covering the following, namely: i) to add a long-term stable power supply in the receiver, ii) customization of receiver command settings, iii) to add a GSM modem and internet protocol for data communication from the receiver to a cloud server, iv) installation of SNIP software in a cloud server to provide CORS corrections with NTRIP caster, and v) testing the NTRIP client performance in the rover receiver. Results from the land cadastre surveys carried out in Bandung, Padang and Tangerang Cities show that the length of time for the fixed positioning with resolved ambiguities could be obtained within less than 5-7 seconds with the rover locations less than $10 \mathrm{~km}$ from the base station. A longer time to obtain fixed solutions required for the distance at the range of 10 to $20 \mathrm{~km}$, and it is becoming more difficult to get fixed solutions over $20 \mathrm{~km}$. Further developments would be carried out using affordable price GNSS OEM boards provided by different manufacturers.
\end{abstract}

\section{INTRODUCTION}

Indonesia Continuously Operating Reference Station (CORS) Network consists of 150 stations, as of November 2018, is still inadequate to provide centimeter-level accuracy with Real Time Kinematic (RTK) positioning across the Archipelago since the average inter-station distance is of about $500 \mathrm{~km}$. The distance is far from $50 \mathrm{~km}$ idealistic distribution that can provide fixed ambiguity resolution in ionospheric distribution (Chen et al. 2001; Wielgosz et al. 2005; Grejner-Brzezinska et al. 2007), resulting in the users of the Indonesian CORS limited in numbers, namely of about 1,906 users (BIG Outlook, 2017). The number of users would be growing significantly in the future as the CORS distribution is improving and costs of geodetic GNSS receivers could be significantly declining to an affordable price in the future.

Efforts on the development of geodetic GNSS receiver with local content are highly encouraged. The opportunity is growing since many re-known GNSS receivers producers are now shifting to produce OEM boards which can be further developed by third parties to their applications. Access to develop the OEM boards has highly reduced cost and more possibility to provide customization of products to specific applications.

The motivation of the research is to develop CORSenabled GNSS receiver with local contents and affordable price. By providing an affordable CORS base receiver, this strongly supports the acceleration of the Indonesia CORS network densification leading to speed up the utilization of GNSS positioning techniques supporting national program on large-scale mapping from cities to village level. The research is inlined with Indonesia national program on technology independence. 


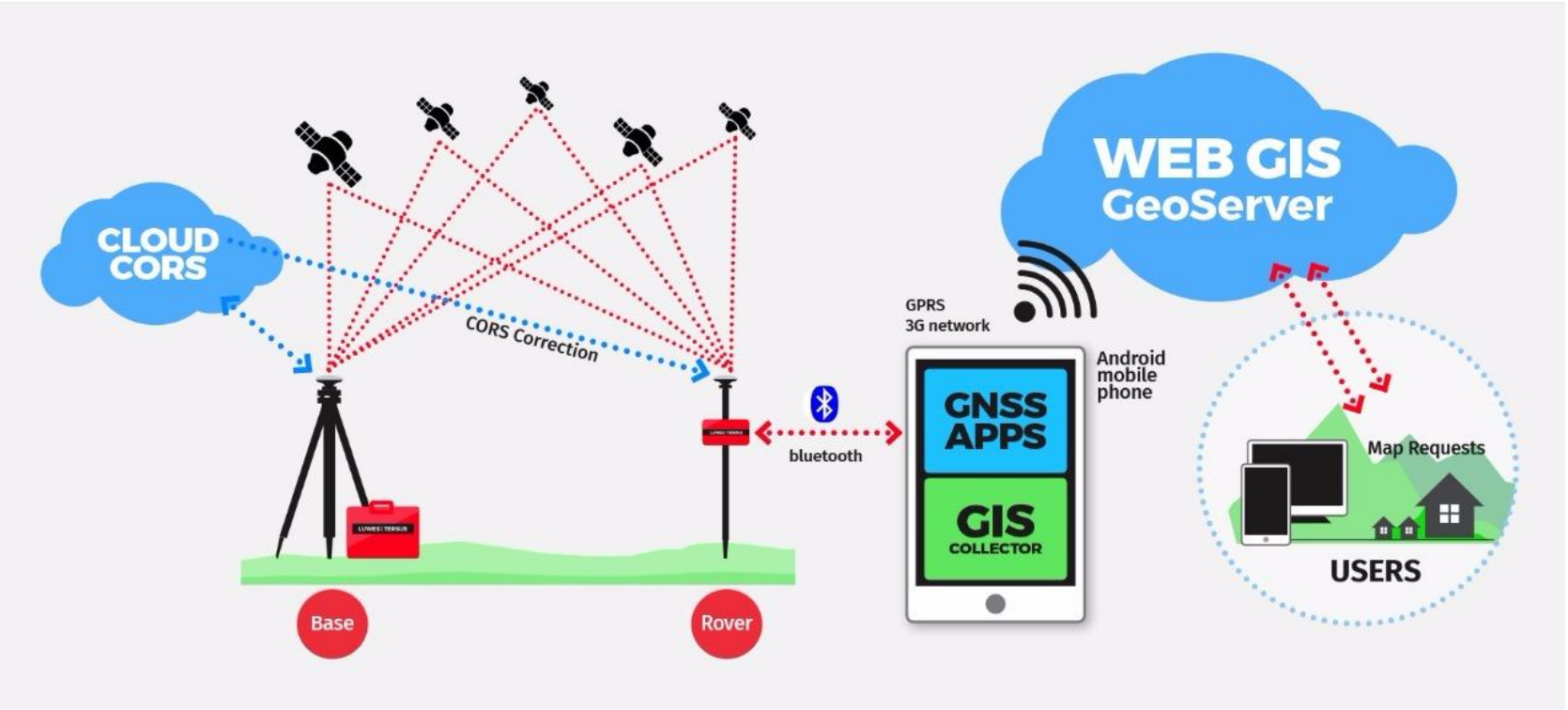

Figure 1 Online Survey Concept

\section{GNSS RECEIVER DEVELOPMENT FOR ONLINE SURVEY}

\subsection{System Design}

An online survey with CORS technique is possible by integrating GNSS positioning with GIS software to the internet of things (IOT) using cellular data communication. The whole system consists of 5 components, namely: i) GNSS Base Receiver, ii) Cloudbased CORS Service, iii) GNSS Rover Receiver, iv) Android Smartphone controller, v) Web GIS Cloud Server. The flow of operation can be described as the following, Figure 1. The base station sends the data to the cloud server which provide service for positioning corrections to the rover via NTRIP Caster. The Android Smartphone controller equipped with NTRIP Client application receives the corrections to compute the point coordinates. The position is stored and displayed using GIS Software in the smartphone. To make the data survey online to internet and access to other partners, the surveyor with the smartphone could send the shapefile periodically to the Web GIS Cloud with GeoServer engine.

\subsection{Base GNSS Receiver}

The OEM board for the base receiver is designed to provide efficient algorithm enabling fast ambiguity resolution within a few seconds. A key element supporting an RTK survey is to select multi-channel receiver which can receive multi-satellite signals namely GPS, BEIDOU, GLONASS and GALILEO. The PCB design should accommodate high quality of electronic components consisting of GNSS OEM boards, cellular modem for data communication, mini PC, power supply system including the electronic casing for protection.
The OEM board selection was carried out based on the data sheet provided by several manufacturers available in the market, namely Tersus, Novatel, Trimble, and CHC. The main criteria that we sought for are the following, namely: i) supporting RTK switch base and rover, ii) option to use Bluetooth connection to Android smartphone, iii) RTK accuracy better than $5 \mathrm{~cm}$, iv) affordable pricing, and v) strong support from the manufacturers for data protocol. Based on the working paper data analysis, it was decided to use the OEM Board Tersus serial BX305.

The GNSS OEM board is integrated with the following components, namely: i) a mini PC for an internal controller, ii) power supply electronic circuit system and UPS, iii) firmware controller and embedded processing, iv) Cellular (GSM) modem dan Bluetooth for data communication. A standard survey grade antenna used is series AX3702. The antenna could be replaced with that of choke ring type registered in International GNSS Service (IGS), if high precision applications are required, as such as monitoring crustal deformation and survey constructions.

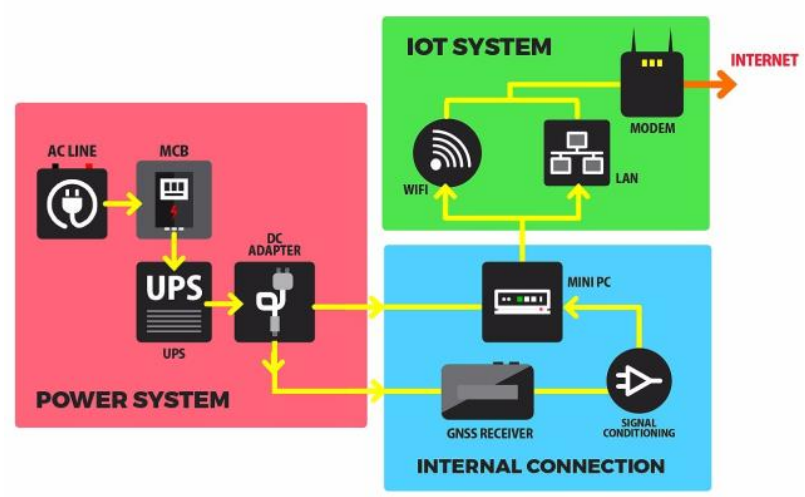

Figure 2 Power System and IOT Feature controlled by mini PC 
A mini PC unit is the system controller embedded to the GNSS OEM board. The conditioning signal is needed to change the GNSS board output data in the form of LVTTL converted into data in the form of USB so that the mini PC can read it. A Mini PC link with a unit power system (UPS) with the capacity of 650KVA for 30 minutes power back up, as shown in Figure 2. A smart power system is highly required to provide control for extreme power flow and to maintain a long time operation and permanent monitoring.

\subsection{Cloud NTRIP Caster}

We decided to use a commercial software namely SNIP, an NTRIP Caster that allows multiple types of data streams, typically containing RTCM corrections messages, to be repeated and sent to various end users over the internet. The advantage of SNIP, it follows multiple industry standards for its message process and connection protocols, allowing any serial port data, any NTRIP Server and client to connect to it. SNIP is now widely used to extend the CORS networks coverage in places not yet served by those of national networks, Figure 3. SNIP provide options to users either using a public NTRIP Caster server namely RTK to Go or a dedicated private server, requiring an extra charge for the service.

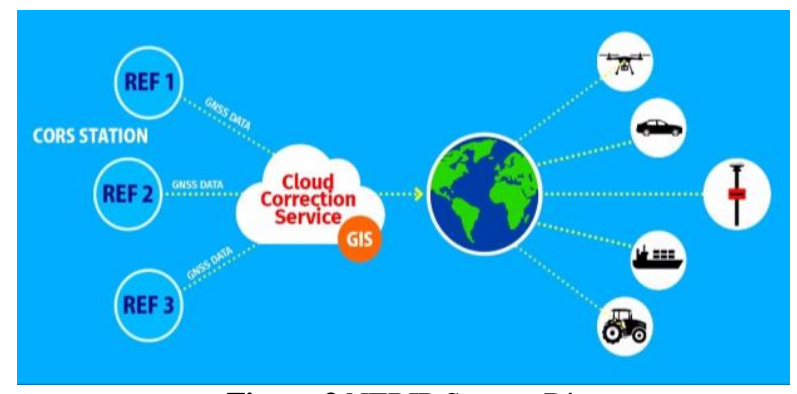

Figure 3 NTRIP System Diagram

\subsection{Rover GNSS Receiver}

The basic design for a rover receiver is to provide a userfriendly receiver that can be easily understood and operated by users with common knowledge on positioning and mapping. The integration of the receiver controller with a GIS collector together in an Android smartphone is a significant step to provide users with a controller of which the users are more familiar than that of a dedicated controller. Mapit GIS software is a simplified GIS command operation that can handle data acquisition with an Android smartphone.

Criteria to measure performance metrics of rover receiver is based on four factors, U.S Department of Defense (2008), namely i) accuracy, ii) time to first fix, iii) continuity, and iv) availability. The accuracy is defined as the difference of the calculated position solution and the true position. Time to First Fix (TTFF) refers to a receiver's state when it has derived and is reporting a valid position solution after being powered.
TTFF is a performance metric of receiver initialization. It is the amount of time that it takes to report a solution from a cold start. Continuity is the likelihood of a detected, but unscheduled navigation function interruption after an operation has been initiated. A loss of RTK lock is a loss of continuity. The RTK availability is defined as the total amount of time as a percentage that a receiver spends in an RTK position solution. An ideal receiver will have an RTK availability close to $100 \%$.

\subsection{Android Smartphone Controller}

Technological development has been significantly turned the Android smartphone as a GNSS controller. This standard feature available in the market is more reliable and even more affordable than that of the controller made specifically to the receiver. Smartphones are becoming more potent with reinforced processors, larger storage capacities, and more data communication methods. An open-source platform Android is commonly used in smartphones, equipped with software package consisting of an operating system, middleware layer, and core applications, Yan and Shi (2013). Bluetooth facilitates data communication between the smartphone and the GNSS Receiver. Bluetooth technology and other similar techniques, with a dramatic increase in Smartphone users, smartphones have gradually turned into an allpurpose portable device.

A smartphone is treated as the external brain of GNSS receiver by adding three main functions as the following: i) Controlling the receiver, ii) Connecting the receiver with CORS Service using NTRIP Client, iii) collecting data and display with GIS Collector. The smartphone device enables the system connected to the external world by facilitating the smartphone to send GIS shapefiles to Web-GIS server allowing multiple users to access data from the field. This achievement is a new state of the art survey with online mode.

\subsection{Web GIS Cloud: GeoServer}

GeoServer is a Java-based software server that allows users to view and edit geospatial data and allows for great flexibility in map creation and data sharing. This uses the Web Feature Service (WFS) standard, which permits the actual sharing and editing of the data that is used to generate the maps, Henderson (2014). GeoServer comes with a WMS that can fit into other Java applications like OpenLayers and can create maps of different formats. GeoServer can display data on any popular modern map applications as such as Google, Yahoo, and Bing. It can also connect to the traditional desktop GIS tools like ESRI ArcGIS. 


\section{RESULT}

There are four testing schemes conducted to measure the performance of the solutions starting from testing the base and rover receivers to the cloud GIS GeoServer.
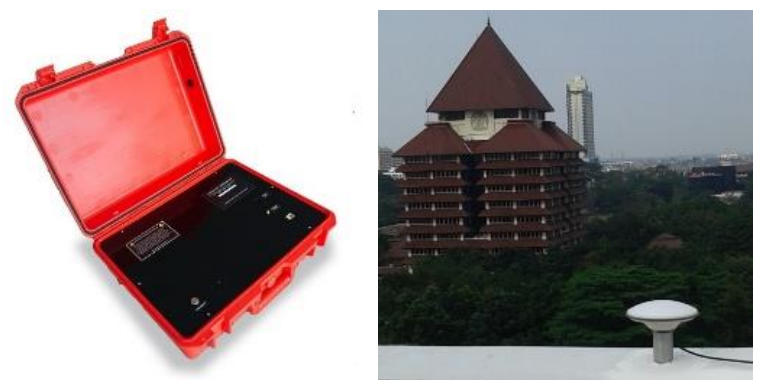

Figure 4 Mobile CORS base station \& Antenna located in the University of Indonesia Campus.

First, testing the mobile GNSS base receiver was carried out by deploying the instrument located in the rooftop of Multidisciplinary Building at the University of Indonesia Campus in Depok City, Figure 4. Data communication between the base station to the cloud CORS service is carried out using a broadband cellular modem. A survey grade antenna with a serial type of AX3702 is installed on the top of the building wall. The performance of the base station has shown a significant improvement to provide CORS service for high precision RTK solution in the surrounding area around the radius of $20 \mathrm{~km}$.

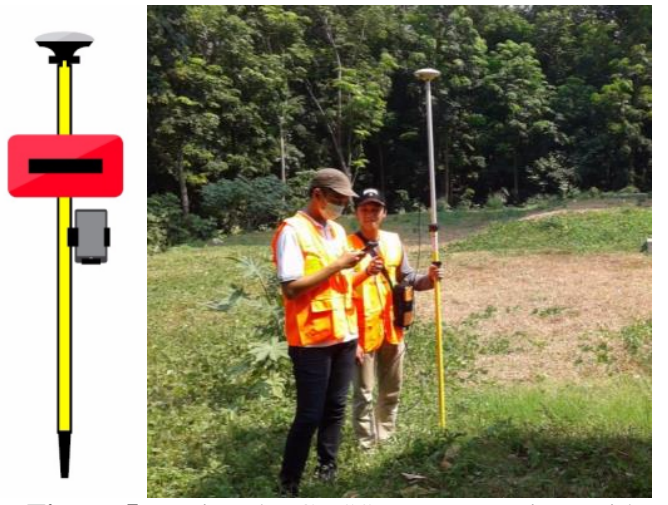

Figure 5 Testing the GNSS Rover Receiver with an antenna pole

Second, testing the performance of rover receiver, as shown in Figure 5, and the NTRIP Caster was carried out in every $5 \mathrm{~km}$ radius from the base station to the maximum distance which can be possible for the rover to resolve ambiguities, as shown in Figure 6. The Table shows that time for fixing ambiguities increases as the location of the rover to the base station is more significant. However, the result shows that time to fixed ambiguities on average is less than 5 seconds within the maximum distance of $20 \mathrm{~km}$. Resolving ambiguities for more than $20 \mathrm{~km}$ is becoming harder and uncertain since the model in the SNIP Caster does not take into account a complicated situation of atmospheric condition.

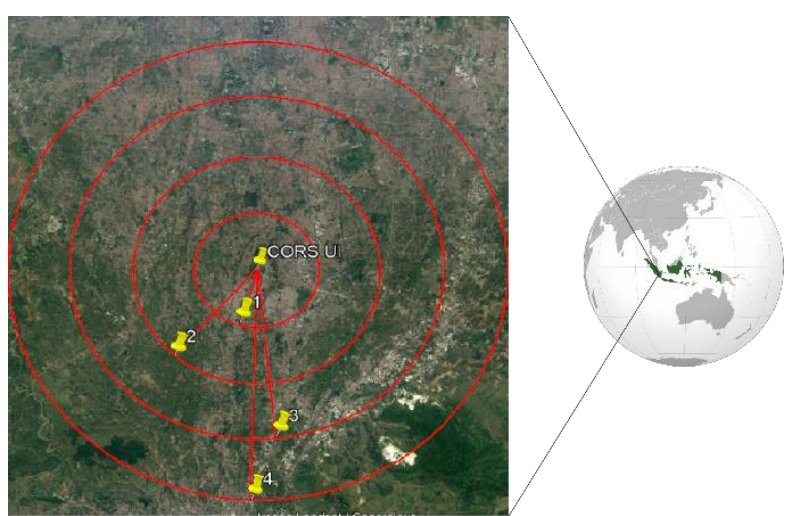

Figure 6 The area test with the base station located in the University of Indonesia Campus. The red circles show the rover distance from the base with $5 \mathrm{~km}$ radius interval.

Third, testing the system by making the distance between the base and rover receivers closer at a distance between $15 \mathrm{~km}$, it has shown a significant improvement to resolve ambiguity to less than 5 seconds. Results from the land cadastre surveys carried out in three big cities, namely Bandung (West Java Province), Padang (West Sumatra Province), and Tangerang (Banten Province), these show that the length of time for the fixed positioning with resolved ambiguities could be obtained within less than 5 seconds with the rover locations less than $10 \mathrm{~km}$ from the base station.

Table 1 Time to acquire for a fixed solution using a base station located in the University of Indonesia

\begin{tabular}{|l|c|c|c|c|c|}
\hline \multicolumn{2}{|l|}{ Point } & 1 & 2 & 3 & 4 \\
\hline $\begin{array}{l}\text { Distance to the base station } \\
(\mathrm{km})\end{array}$ & 5 & 10 & 15 & 20 \\
\hline \multirow{5}{*}{$\begin{array}{l}\text { Time for a fixed } \\
\text { solution (second) }\end{array}$} & P1 & 2 & 3 & 5 & 5 \\
\cline { 2 - 6 } & P3 & 2 & 2 & 4 & 4 \\
\cline { 2 - 6 } & P4 & 1 & 2 & 2 & 5 \\
\cline { 2 - 6 } & P5 & 1 & 2 & 3 & 2 \\
\cline { 2 - 6 } & P6 & 1 & 1 & 1 & 4 \\
\cline { 2 - 6 } & P7 & 1 & 2 & 2 & 5 \\
\cline { 2 - 6 } & P8 & 2 & 2 & 1 & 2 \\
\hline & P9 & 1 & 2 & 1 & 3 \\
\hline & P10 & 2 & 2 & 3 & 2 \\
\hline & P11 & 1 & 2 & 2 & 3 \\
\hline Average Time & 1,45 & 2 & 2,55 & 3,55 \\
\hline
\end{tabular}




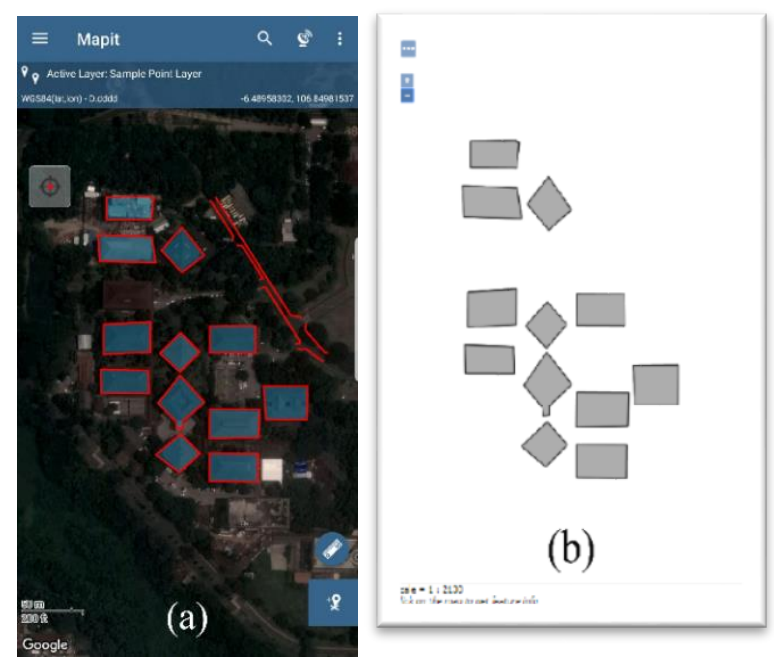

Figure 7 The measured area displayed in the Smartphone collector (a) and presented data in web GIS (b).

Finally, testing for the sending of the GIS data layers collected in the Android smartphone to the web GIS GeoServer was carried out by doing field data collections in the University of Indonesia Campus. The survey target is to map around 11 buildings in the campus as shown in the Mapit GIS Collector, Figure 7 (a). The time for data collecting was completed around two hours only. Then the shapefile of the sampling was sent via cell phone to the Web GIS and displayed by GeoServer as shown in Figure 7 (b).

\section{CONCLUSION}

The objective of the paper is to develop a GNSS CORS receiver which is easy to deploy in the field as a base station and to provide RTK correction services to rover receivers using a cloud server. The system has been successfully developed to make possible an online survey by combining high precision RTK CORS positioning with a web GIS cloud. This technique was tested resulting in less than 5 seconds for ambiguity resolution with inter-station distances of maximum 20 $\mathrm{km}$ and the accuracy level between $2-5 \mathrm{~cm}$. There are four essential elements to improve the CORS positioning technique into online mode, namely: i) a base GNSS receiver connected to the cloud server caster, ii) a rover GNSS receiver equipped with an Android smartphones controller of which the GIS collector is installed, iii) the cellular coverage for strong data connection to IOT, and iv) a web GIS cloud with GeoServer for online map creation and data sharing. The online survey solution demonstrates a significant improvement to a faster field data acquisition and GIS data sharing.

\section{ACKNOWLEDGMENT}

This development was financed by PT Luwes Inovasi Mandiri; a start-up company focused on solution development for precise GNSS positioning. Support provided by the Department of Geoscience of University of Indonesia and Badan Informasi Geospasial (BIG) during the project is gratefully acknowledged.

\section{REFERENCES}

Badan Informasi Geospasial (BIG) (2017). Outlook Pusat Jaring Kontrol Geodesi dan Geodinamika 2017. Cibinong.

Chen W, Hu C, Chen Y, Ding X (2001) Rapid static and kinematic positioning with HongKong GPS active network. In: ION GPS 2001, Salt Lake City, UT, pp 346-352

Grejner-Brzezinska D, Kashani I, Wielgosz P, Smith D, Spencer P, Robertson D, Mader G (2007) Efficiency and reliability of ambiguity resolution in network-based real-time kinematic GPS. J Surv Eng 133(2):56-65

Henderson, Colin (2014). Mastering Geoserver. Birmingham, UK.

Herring, T. A., R. W. King, M. A. Floyd, S. C. McClusky (2015). Introduction to GAMIT/GLOBK. Department of Earth, Atmospheric, and Planetary Sciences Massachusetts Institute of Technology, Cambridge, MA 02139, USA.

Schwieger, V., Lilje, M., \& Sarib, R. (2009). GNSS CORS Reference Frames and Services. In 7th FIG Regional Conference, Hanoi, Vietnam (Vol. 19, No. 22.10, p. 2009).

Snay, R. A., \& Soler, T. (2008). Continuously operating reference station (CORS): history, applications, and future enhancements. Journal of Surveying Engineering, 134(4), 95-104.

SNIP. (2018). About SNIP. Cited in https://www.usesnip.com/ [14 Agustus 2018]

Takasu, T., \& Yasuda, A. (2009). Development of the low-cost RTK-GPS receiver with an open source program package RTKLIB. In International Symposium on GPS/GNSS (pp. 4-6). International Convention Center Jeju Korea.

Tersus GNSS Inc, (2016). User Manual Precis BX305 Precise GNSS RTK Board. Cited in https://www.tersus-gnss.com/ [8 December 2018]

U.S. Department of Defence. (2008). Global Positioning System Standard Positioning Service. Retrieved from http://www.gps.gov/technical/ps/2008-SPSperformance-standard.pdf

Wielgosz P., Kashani I, Grejner-Brzezinska D (2005) Analysis of long-range network RTK during severe ionospheric storm. Journal of Geodesy 79(9):524531

Yan, M., and Shi, H (2013). Smart Living Using Bluetooth Based Android Smartphone. International Journal of Wireless \& Mobile Networks (IJWMN) Vol. 5, No. 1, February 2013 